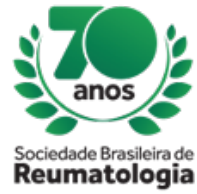

\title{
INFLAMMATORY GIANT GASTRIC ULCERS DUE TO POLYARTERITIS NODOSA ACTIVITY
}

Gisela Rios Machado (Hospital das Clínicas- FMUSP, São Paulo, SP, Brasil), Lorena Elizabeth Betancourt (Hospital das Clínicas-FMUSP, São Paulo, SP, Brasil), Luciana Parente Costa Seguro (Hospital das ClínicasFMUSP, São Paulo, SP, Brasil), Lissiane Karina Noronha Guedes (Hospital das Clínicas-FMUSP, São Paulo, SP, Brasil), Eduardo Ferreira Borba (Hospital das Clínicas-FMUSP, São Paulo, SP, Brasil), Bruna Giusto Bunjes (Hospital das Clínicas-FMUSP, São Paulo, SP, Brasil), Glaucia Ferreira Abrahão (Hospital das Clínicas-FMUSP, São Paulo, SP, Brasil), Felipe Freire Silva (Hospital das Clínicas-FMUSP, São Paulo, SP, Brasil), Rosa Maria Rodrigues Pereira (Hospital das Clínicas-FMUSP, São Paulo, SP, Brasil)

\section{BACKGROUND}

Polyarteritis nodosa (PAN) is a vasculitis characterized by necrotizing inflammation that can affect small and medium vessels with multisystemic involvement. Gastrointestinal manifestations including abdominal pain, nausea and vomiting are reported in up to $35 \%$ of cases and may be related to mesenteric arteritis. We report a case of inflammatory giant gastric ulcers as a manifestation of the disease, with a good response to immunosuppressive treatment.

\section{CASE REPORT}

A 29-year-old male patient with a 3-month history of fever, weight loss of $24 \mathrm{~kg}$, epigastric pain, nausea, vomiting, purpuric painful lesions in the lower limbs, and paresthesia in the hands. Laboratory test showed normocytic anemia, thrombocytocis and elevated C-reactive protein (CRP, $431 \mathrm{mg} / \mathrm{L}$ ) and erythrocyte sedimentation rate (ESR, 89mm). Tests for antinuclear factor (ANA), antineutrophil cytoplasmic antibody (ANCA), antiphospholipid antibodies and cryoglobulins were negative, with low titer rheumatoid factor $(22.5 \mathrm{UI} / \mathrm{ml})$. Viral serologies were negative. Upper gastrointestinal endoscopy (EGD) showed multiple giant gastric ulcers ( 4 to $6 \mathrm{~cm}$ ). Biopsy of gastric ulcers showed intense active chronic gastritis, with no evidence of malignancy, and negative tests for Helicobacter pylori, cytomegalovirus, herpes virus and acid-alcohol fast bacilli. Gastrinoma, inflammatory bowel disease and hyperparathyroidism were excluded. Biopsy of skin purpuric lesions showed dermal and hypodermal vasculitis. Electroneuromyography revealed axonal peripheral neuropathy of the left lower limb. Patient was diagnosed with PAN and was treated with intravenous methylprednisone pulses $(1,000 \mathrm{mg} /$ day for 3 days $)$ and cyclophosphamide ( $1 \mathrm{~g} /$ month for 6 months). Patient presented significant improvement of the clinical condition, with resolution of symptoms and normalization of CRP and ESR. Control EGD demonstrated cicatricial lesions with mild chronic gastritis. Patient discontinued treatment and lost follow-up, with reactivation of the disease after 3 years with the same dyspeptic symptoms, elevated CRP and ESR, giant gastric ulcers on EGD, which improved after immunosuppressive treatment.

\section{CONCLUSION}

The authors claim attention to the unusual clinical presentation of inflammatory giant gastrointestinal ulcers as a manifestation of polyarteritis nodosa activity, which improved after high dose glucocorticoid and immunosuppressive treatment. 